TITLE:

\title{
A high-resolution small-angle light scattering instrument for soft matter studies
}

\section{$\operatorname{AUTHOR}(\mathrm{S})$ :}

Nishida, Koji; Ogawa, Hiroki; Matsuba, Go; Konishi, Takashi; Kanaya, Toshiji

\section{CITATION:}

Nishida, Koji ... [et al]. A high-resolution small-angle light scattering instrument for soft matter studies. Journal of Applied Crystallography 2008, 41(4): 723-728

\section{ISSUE DATE:}

2008-08

URL:

http://hdl.handle.net/2433/93445

\section{RIGHT:}

Copyright 2008 (c) International Union of Crystallography; This is not the published version. Please cite only the published version.; この論文は 出版社版でありません。引用の際には出版社版をご確認ご利用くださ い。 


\title{
High Resolution Small-Angle Light Scattering Instrument for Soft Matter Study
}

Koji Nishida*, Hiroki Ogawa, Go Matsuba, Takashi Konishi and Toshiji Kanaya

Institute for Chemical Research, Kyoto University, Uji, Kyoto-fu 611-0011, Japan. Correspondence e-mail: knishida@scl.kyoto-u.ac.jp

Synopsis A small-angle light scattering (SALS) instrument with a high resolution at low angles and a high signal-to-noise ratio, which is suitable for soft matter study, has been developed.

\begin{abstract}
A small-angle light scattering (SALS) instrument with a high resolution at low angles and a high signal-to-noise ratio has been developed. Both a wide dynamic range and a wide scattering vector range are achieved using a two-dimensional array of complementary metal oxide semiconductor (CMOS) image sensors. These instrument characteristics have enabled us to obtain high quality light scattering data from soft matter systems. This set-up is especially well suited to studies of systems with a weak scattering power and/or a timedependent structure evolution in a wide spatial range ranging from sub-micrometer to submillimeter. An application of this instrument to a polyelectrolyte blend and an extremely thin blend film are reported.
\end{abstract}

Keywords: small-angle light scattering (SALS); complementary metal oxide semiconductor (CMOS) image sensor; soft matter 


\section{Introduction}

When the light scattering (LS) technique was invented, its major application to polymers was the characterization of a polymer chain in solution, such as extraction of a molecular weight and a radius of gyration (Debye, 1947; Zimm 1948-1). Initially commercial instruments for the light scattering measurements were of course not available and the instrument optics was custom designed by researchers (Debye 1946; Zimm 1948-2). In order to extract characteristic quantities for polymer chains one should scan mainly a wide scattering angle range. Hence the instruments were usually equipped with a photometer positioned on a wideangle goniometer. This type of light scattering technique has been perfected for half a century and is well established (Chu, 1991). Nowadays several manufacturers offer instruments which are also equipped with a correlator used for the dynamic light scattering measurements.

As the light scattering technique was maturing, it had been applied to investigate highly ordered structures in the bulk, especially the spherulite structure of crystalline polymers (Plaza \& Stein, 1959). In order to investigate structures which are optically anisotropic a depolarized light scattering (DPLS) technique was employed. In DPLS a polarizer and an analyzer are inserted before and after the sample position. Since the higher order structure is large compared with the molecular dimension, the small scattering angle range is scanned. Therefore this type of light scattering technique is often termed the small-angle light scattering (SALS) or low-angle light scattering (LALS) in order to distinguish it from the original (wide scattering angle) light scattering.

In the early days of SALS technique an arc lamp and a photographic film were used as the light source and the detector for the scattered light, respectively (Plaza \& Stein, 1959; Stein \& Rhodes, 1960). In order to select a beam of parallel and monochromatic light from the diverging white light source a lens and pinhole device and monochromatic filters were employed (Plaza \& Stein, 1959). Somewhat later, a He-Ne laser was introduced as the source of the coherent light (Samuels, 1966). A photomultiplier tube installed on a small-angle goniometer was also used in addition to the photographic film to aid a quantitative angular profile analysis. The introduction of the laser as the light source was a breakthrough in the development of the SALS. As the devices that select a beam of parallel and monochromatic light became redundant more researchers were able to construct their own SALS instruments.

The photodiode array made it possible to measure the whole angular profile rather than scanning the angular range. This has lead to the time-resolved measurements (Okada et al., 1992). In step with the development of cameras for consumer use, the photographic film was replaced by a charge-coupled device (CCD) image sensor to record two-dimensional SALS data. However, the exposure area of the CCD image sensor that is available at a reasonable 
price is usually very small. Accordingly to do measurements with a limited exposure area $\mathrm{CCD}$ a condenser lens is necessary. This lens converges the scattered light on the CCD plane (Ferri, 1997; Kawai \& Strobl, 2004). Alternatively, the scattering profile projected on a white screen is indirectly recorded by a CCD camera equipped with an imaging lens (Fukushima et al., 2005). These above two methods have both advantages and disadvantages. A merit of the former method is that every scattered amount of light can be directly brought into the CCD plane without a loss of intensity. However it is necessary, in order to focus the scattering image on the CCD plane, to keep the distances of the sample to the condenser lens and the condenser lens to the CCD plane fairly rigid (as dictated by the focal length (F) of the condenser lens). This means there is no flexibility in the camera length in this scattering geometry. The latter method (indirect recording) gives flexibility of adjusting the camera length, but inevitably one loses the brightness and resolution due to the smearing effect on the screen when the scattered light is projected. In the end, the image sensor having a large exposure area is considered to be an ideal detector for SALS.

In recent years, CCD image sensors and a complementary metal oxide semiconductor (CMOS) image sensors, both of which have a large exposure area, became available. It is true that the former, especially a cooled type, gives access to about one order of magnitude wider dynamic range than the latter. Regrettably though, a CCD image sensor is still much more expensive than the CMOS image sensor. Thus we have designed a SALS instrument equipped with a CMOS image sensor having a large exposure area as the detector. In order to utilize the full dynamic range of this sensor we have optimized the optics of our SALS instrument to suppress the noise level as much as possible. In this study we describe the basic performance of this instrument as well as some applications to soft matter studies.

\section{Instrumental setup}

The optical geometry of the SALS instrument constructed by us is sketched in Fig. 1. The light source is a $50 \mathrm{~mW}$ He-Ne laser with the wavelength $\lambda=0.6328 \mu \mathrm{m}$ purchased from Showa Optronics Co., Ltd. The beam power is reduced by the neutral density (ND) filters to meet each experimental requirement. Optical components, such as filters and lenses, were produced by SIGMA KOKI Co., Ltd. unless otherwise specified. As mentioned in the Introduction section, a laser source is assumed to give a beam of parallel light rays. This holds in theory but in practice a small amount of unwanted stray light is emitted from the laser-tube window (LW). For a depolarized light scattering (DPLS) measurement, and for a strongly scattering sample, such a small amount of stray light does not matter much. However, it will affect the signal-to noise ratio strongly in a case of a weakly scattering sample. In order to eliminate the stray light, a pair of achromatic lenses $\left(\mathrm{L}_{1}\right.$ and $\left.\mathrm{L}_{2}\right)$ and an 
aperture (A) are employed. This pair of lenses acts as a filter, and is also used to reduce the beam diameter. The distances of $\mathrm{L}_{1}$ to $\mathrm{A}(=200 \mathrm{~mm})$ and $\mathrm{A}$ to $\mathrm{L}_{2}(=100 \mathrm{~mm})$ give the desired effect. This is a necessary precaution since a small hole (diameter $=2.2 \mathrm{~mm}$ ) in a temperature-controlled stage for samples (S) would become another source of the stray light if the incident beam interferes with it.

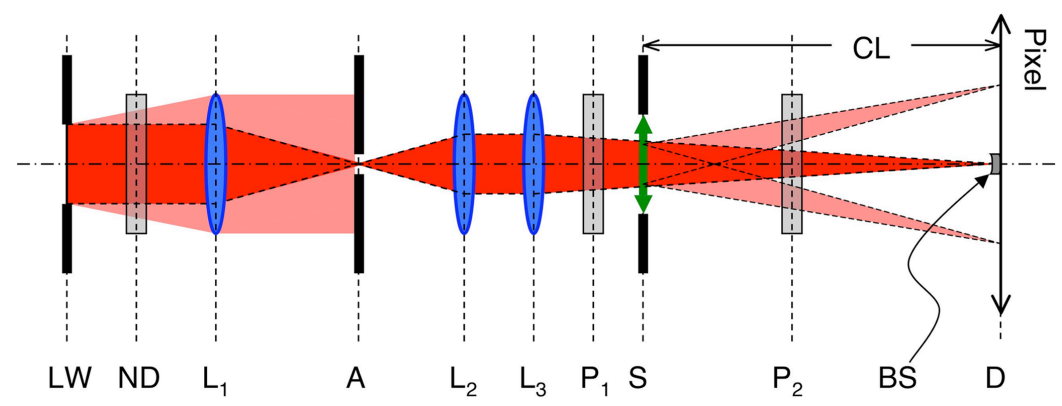

Figure 1 Schematic diagram of the optical geometry of the SALS instrument. LW: laser-tube window, ND: neutral density filters, $\mathrm{L}_{1}$ : lens $\left(\mathrm{F}_{1}=200 \mathrm{~mm}\right), \mathrm{A}$ : aperture (diameter $\left.=0.3 \mathrm{~mm}\right), \mathrm{L}_{2}$ : lens $\left(\mathrm{F}_{2}=100 \mathrm{~mm}\right), \mathrm{L}_{3}$ : lens $\left(\mathrm{F}_{3}=450 \mathrm{~mm}\right), \mathrm{P}_{1}$ : polarizer, $\mathrm{S}$ : sample, $\mathrm{P}_{2}$ : analyzer, BS: beam stopper (semitransparent), D: detector plane, and CL: camera length.

Subsequently the incident beam is focused on the detector plane (D) by a lens $\mathrm{L}_{3}$. The sample position (S) can be selected between $\mathrm{L}_{3}$ and $\mathrm{D}$, unless the temperature-controlled stage interferes mechanically with $\mathrm{L}_{3}$ or $\mathrm{D}$. Thus the sample to detector distance, or the camera length $(\mathrm{CL})$, is flexible fulfilling different experimental conditions. The longest and the shortest CLs of this instrumental setup are $400 \mathrm{~mm}$ and $100 \mathrm{~mm}$, respectively. The incident beam is finally screened by a beam stopper (BS) placed just before the detector D. Since A and D are optically conjugated, the stray light that is projected on D should be contained within the shaded area of A (see Figure 1 for reference). However, in practice the diameter of the beam stop BS would better be selected according to a type of experiment desired. In the present instrument a BS with $1.5 \mathrm{~mm}$ diameter usually gives reasonable data up to its edge (see section 3.1). In this case, the lowest accessible $q$ is $0.02 \mu \mathrm{m}^{-1}$ for the longest camera length $(\mathrm{CL}=400 \mathrm{~mm})$ as is shown later. When a sample has a very weak scattering power, a BS with $4.0 \mathrm{~mm}$ diameter is used. This diameter is generous since it would cover any leakage of the direct beam. In this case, the lowest accessible $q$ is $0.056 \mu \mathrm{m}^{-1}$ for the longest CL.

If the monitoring of the transmittance of the sample is necessary, especially in the timeevolving measurements, the center part of the BS is replaced by a semi-transparent material (exposed negative film). The intensity of the transmitted light is monitored by the pixels of the sensor placed just behind the center part of the BS. Thus the measurements of the scattering and the transmittance can be conducted simultaneously. For the DPLS 
measurements, a polarizer $\left(\mathrm{P}_{1}\right)$ and an analyzer $\left(\mathrm{P}_{2}\right)$ are inserted before and after the specimen, respectively.

A CMOS image sensor (Rad-icon Imaging Corp.) is used as the detector in this study. The CMOS image sensor consists of $2048 \times 1024$ pixels and the each pixel covers the area of 48 $\mu \mathrm{m} \times 48 \mu \mathrm{m}$; hence an area of ca. $10 \mathrm{~cm} \times 5 \mathrm{~cm}$ is fully covered. The reported nominal dynamic range of the sensor is 12 bit $(=4096)$. The time necessary to save one scattering image using the provided software and a PC with a $2.8 \mathrm{GHz}$ Pentium 4 processor was $0.5 \mathrm{sec}$ without binning the pixels. This is the minimal time resolution in the time-evolving measurements at present. This value will be reduced to nominally $0.37 \mathrm{sec}$ if a PC with higher specification is used.

\section{Basic characteristics of the instrument}

\subsection{Beam profile}

Fig. 2(a) shows a two-dimensional beam profile recorded on the detector plane (D) without a beam stopper. The profiles in the vicinity of the beam center are enlarged (see the scale). Fig. 2(b) displays a one-dimensional beam profile along the equator line that passes through the highest intensity point. The solid curve represents a Gaussian distribution fitted to this profile.
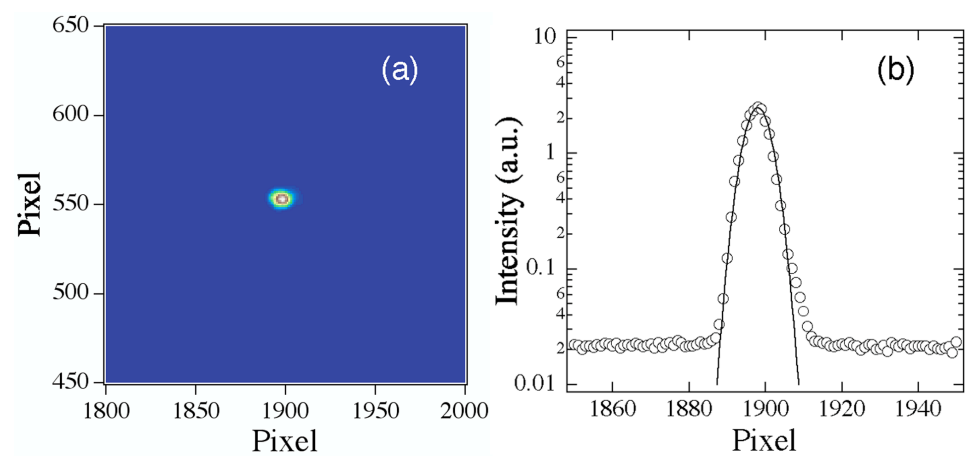

Figure 2 Two-dimensional (a) and a one-dimensional (b) beam profiles on the detector plane without a beam stopper. One pixel corresponds to $48 \mu \mathrm{m}$.

As seen from Fig. 2(b), the full width at half maximum is ca. 8 pixels. This pixel value corresponds to $384(=8 \times 48) \mu \mathrm{m}$ in real space; it also corresponds to $0.0048 \mu \mathrm{m}^{-1}$ in the absolute value of the scattering vector $q(=4 \pi \sin \theta / \lambda$, where $\theta$ and $\lambda$ being the scattering angle and wave length, respectively) when the longest camera length ( $\mathrm{CL}=400 \mathrm{~mm})$ is selected. Hence this $q$ value corresponds to the full limit of the $q$ resolution of the present 
instrumental set-up. The actual $q$ resolution will be discussed later. The base width where the intensity is comparable to the level of a base line is ca. 30 pixels. This pixel value corresponds to $1.44 \mathrm{~mm}$. Hence the beam stop BS with a diameter of $1.5 \mathrm{~mm}(>1.44 \mathrm{~mm})$, as discussed above, is considered to be a reasonable choice.

\subsection{Dynamic range and linearity of detector}

Although the nominal dynamic range of the sensor itself is 12 bit, the effective dynamic range that can be used for the data analysis depends on the type of experiment. Therefore we have estimated a practical dynamic range of the present SALS instrument in terms of diffraction from a pinhole. The Fraunhofer diffraction from a pinhole, the so-called Airy pattern, is given by (Hulst, 1957):

$$
I(x)=\left[2 J_{1}(x) / x\right]^{2}
$$

where $J_{1}(x)$ is the Bessel function of the first kind, and $x$ is a product of $q$ and the radius of the pinhole $R(x=q R)$. Since the envelope curve for the Airy function behaves as (-3) power of $q$, a pinhole is often used as a standard sample to test SALS instruments (Ferri, 1997).

Fig. 3 shows a two-dimensional scattering image from a pinhole with a $50 \mu \mathrm{m}$ diameter. Concentric periodic rings are clearly visible. A bright innermost ring adjacent to the shadow of the beam stopper is the 0 -th diffraction peak centered on $q=0$. The first and the higher order diffraction peaks from the $50 \mu \mathrm{m}$ pinhole are perfectly resolved.

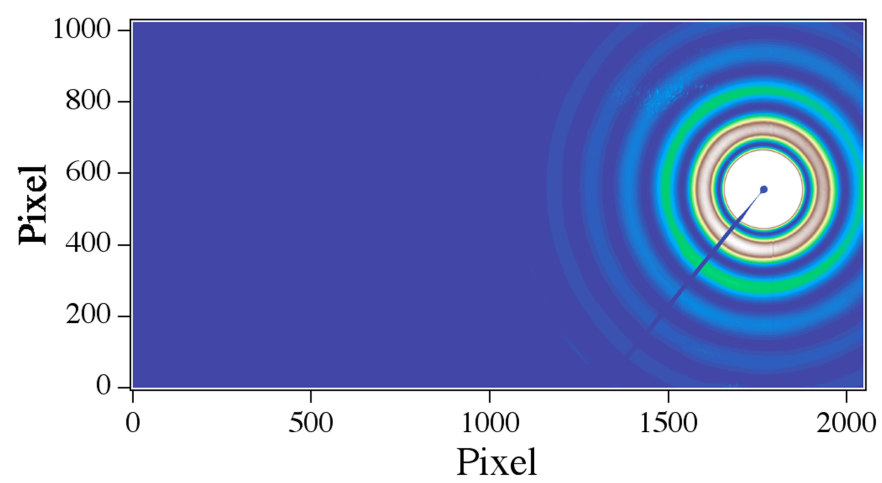

Figure 3 Two-dimensional scattering image from a pinhole with $50 \mu \mathrm{m}$ in diameter for $\mathrm{CL}=400$ $\mathrm{mm}$. One pixel corresponds to $48 \mu \mathrm{m}$.

In order to evaluate the diffraction profile quantitatively, the two-dimensional scattering image is circularly averaged and converted into a one-dimensional intensity profile. Fig. 4(a) 
shows the one-dimensional intensity profile taken at a single attenuation level. The cubic cosine correction for the incident angle was carried out and the electronic noise, which was measured without beam, was subtracted. However, the correction for the sensitivity of the image sensor was omitted. The attenuation level is intentionally selected in order to saturate the counting capacity of the sensor. Accordingly, the intense part, i.e. the low $q$ region, of the intensity profile has a plateau at $4 \times 10^{3}$ on the given scale. On the other hand, the intensity in the highest $q$ region is ca. $2 \times 10^{0}$. The envelope curve for the measured diffraction peaks reproduces the (-3) power of $q$ fairly well. Therefore, the practical dynamic range at a single attenuation level is evaluated as ca. $2 \times 10^{3}$ at least. The overall linearity of the sensor is also fairly well reproduced (with three digits precision) without the correction for the sensitivity, as the intensity can be described by the power law.
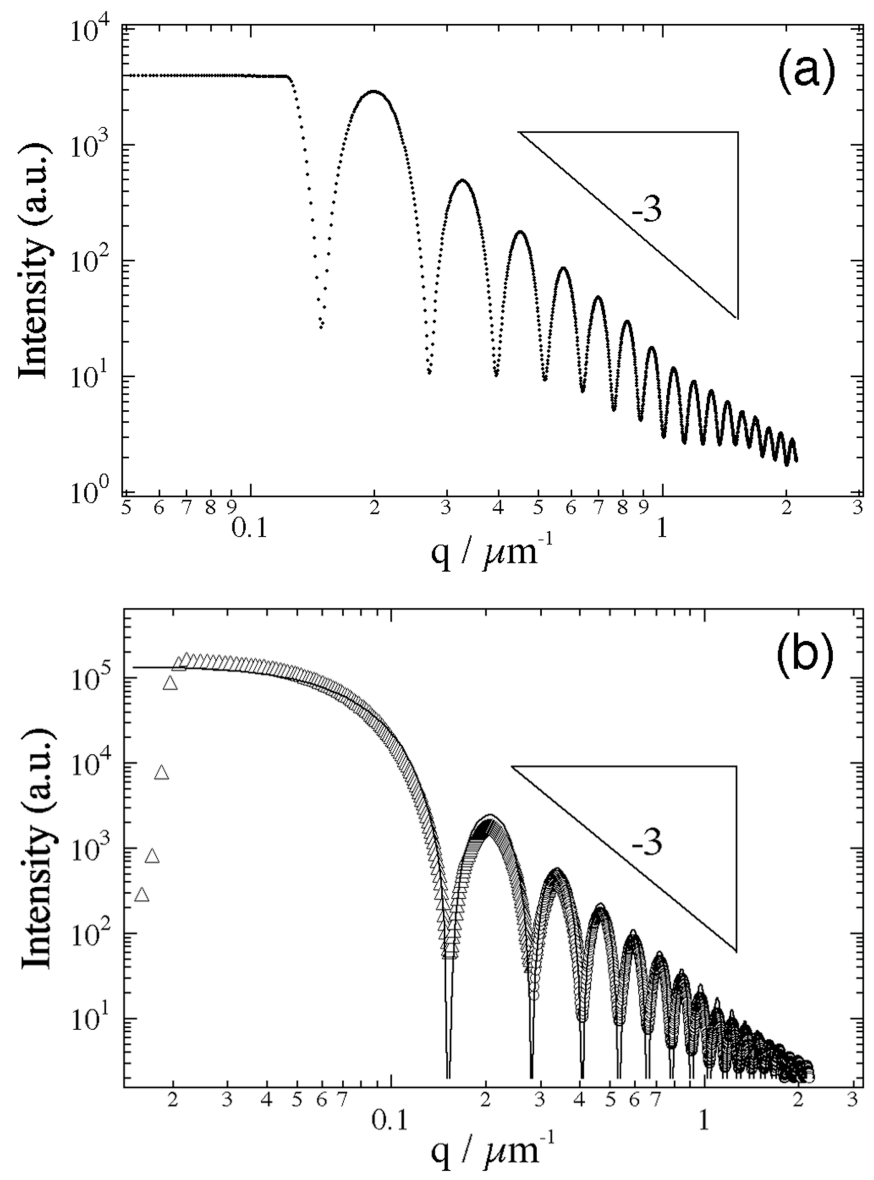

Figure 4 (a): One-dimensional intensity profile of the scattering from a pinhole with $50 \mu \mathrm{m}$ in diameter taken at a single attenuation level. (b): combined profile using two data sets taken at two different attenuation levels. The solid curve represents the Airy function.

To meet a need for the wider dynamic range, multiple data sets taken at different attenuation levels are usually combined. Fig. 4(b) shows such an example, where two data 
sets taken at two different attenuation levels are combined by overlapping the 2-nd diffraction peak. The relative attenuation factor for the data set of the higher intensity part was $1 / 60$. The combined diffraction profile reproduces well the Airy function to five digits, except for the narrow minima, which are somewhat smeared due to the resolution. In addition to the wide dynamic rage, a very wide scattering vector range from $q=0.02 \mu \mathrm{m}^{-1}$ to $2.2 \mu \mathrm{m}^{-1}$, i.e. two orders of magnitude, is covered with a single camera length $(\mathrm{CL}=400 \mathrm{~mm})$. The higher limit of the scattering vector range can be extended to ca. $q=8 \mu \mathrm{m}^{-1}$ by using the shortest camera length $(\mathrm{CL}=100 \mathrm{~mm})$. Then the diffraction from a Bragg spacing down to $0.8 \mu \mathrm{m}$ can be resolved.

\subsection{Resolution of scattering vector}

In order to verify the available $q$ resolution, diffraction patterns from pinholes with rather "macroscopic" sizes were measured. Figs. 5(a) and (b) show the one-dimensional intensity profiles from handmade pinholes with $0.53 \mathrm{~mm}$ and $1.00 \mathrm{~mm}$ diameter, respectively. The diffraction peaks from the pinhole with $0.53 \mathrm{~mm}$ diameter are perfectly resolved. Moreover, the diffraction peaks from the pinhole with $1.00 \mathrm{~mm}$ in diameter are still resolved. Thus the $q$ resolution is guaranteed less than $0.0063(=2 \pi / 1000) \mu \mathrm{m}^{-1}$ in the present set-up. Hence the full limit of the $q$ resolution $\left(0.0048 \mu \mathrm{m}^{-1}\right)$, which was estimated previously from the beam width, is almost attained.
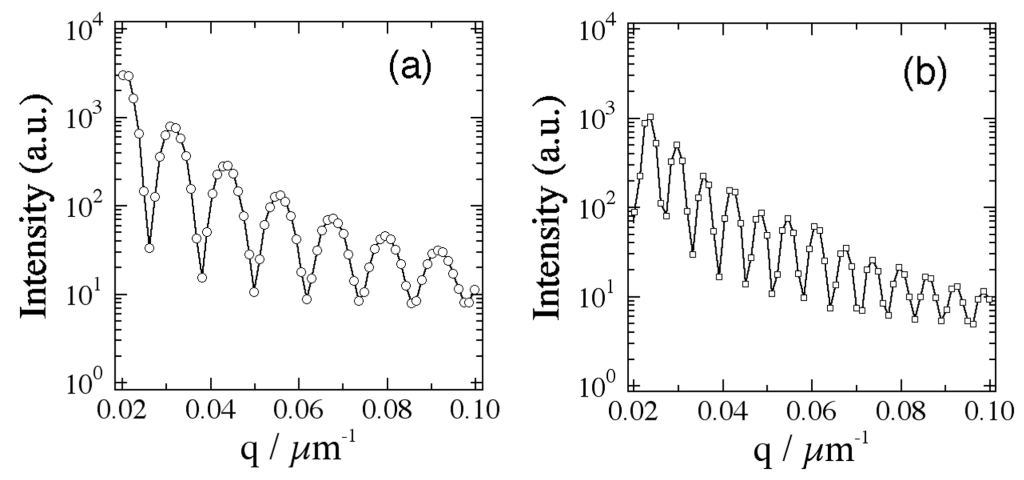

Figure 5 (a): One-dimensional intensity profile of the scattering from a pinhole with $0.53 \mathrm{~mm}$, and (b): with $1.00 \mathrm{~mm}$ in diameter.

\section{Applications to soft matter}

The SALS instrument constructed by us is quite versatile, and we present just a small selection of possible study subjects. One of these is a phase separation in blends, both industrially and academically important field in soft matter studies. The phase separation 
process, especially during the spinodal decomposition, can be described by characteristic power laws in space and time. Hence the observation of the spinodal decomposition in the wide scattering vector and intensity range should be very suitable to test the performance of the SALS instrument.

The first example is the observation of the phase separation process of an aqueous mixture of polyelectrolytes (sodium salt of polyvinylsulfuric acid (NaPVS) and sodium salt of polystyrene sulfonate (NaPSS)). The phase separation was initiated by cooling the sample from $70{ }^{\circ} \mathrm{C}$ to $50{ }^{\circ} \mathrm{C}$, since this system has an upper critical solution temperature (UCST) type phase diagram (Nishida, 2001, Tsubouchi, 2007). Fig. 6 shows the time-resolved onedimensional intensity profiles (after cooling) for the aqueous mixture of $(0.3: 0.7) \mathrm{NaPVS} /$ NaPSS blend and the total concentration of $1.2 \mathrm{~mol} / \mathrm{l}$. Time evolution of the spinodal peak is clearly observed; moreover, the intensity in the $q$ region higher than the position of each peak shows the (-4) power dependence on $q$ with the precision better than two digits in intensity. This is the well known Porod's law which characterizes the domains formed during the phase separation having 3-dimensional smooth surface. Further details of the phase separation behavior of the aqueous mixture of polyelectrolytes using SALS will be reported elsewhere. Judging from the present results our SALS instrument is well suited for studies of the phase separation in 3-dimensional systems.

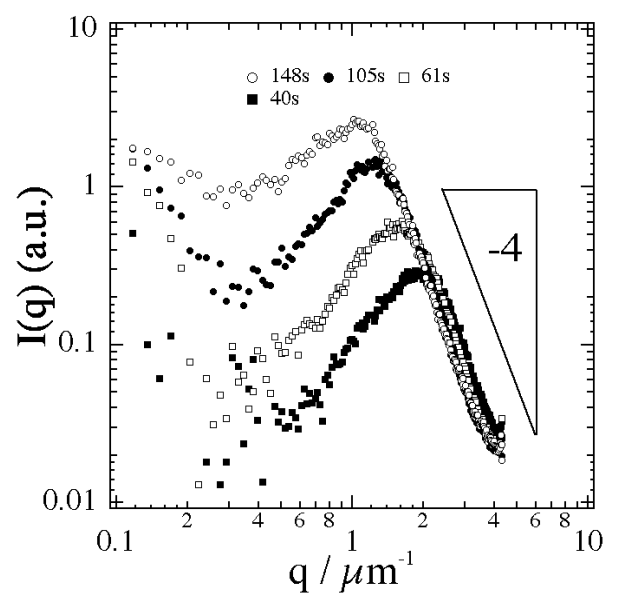

Figure 6 Time-resolved one-dimensional intensity profiles for aqueous mixture of NaPVS / NaPSS blend $\left(0.3: 0.7\right.$ ratio) and the total concentration of $1.2 \mathrm{~mol} / 1$. Spectra taken after cooling from $70{ }^{\circ} \mathrm{C}$ to $50{ }^{\circ} \mathrm{C}$.

The construction rationale behind present SALS instrument was to study the phase separation behavior within extremely thin films as exemplified below. A SALS study on the phase separation between polystyrene (PS) and poly(vinyl methyl ether) (PVME) in the bulk system was reported previously (Hashimoto et al., 1983). However, the phase separation 
behavior of these polymers in the thin film has been unknown, especially when the film thickness is comparable to or less than the characteristic wavelength of the phase separation. Since this system has a lower critical solution temperature (LCST) type phase diagram, the phase separation process was initiated by heating. A snapshot of the two-dimensional scattering image after heating up to $115{ }^{\circ} \mathrm{C}$ is shown in Fig. 7(a). In Fig 7(b) the timeresolved one-dimensional intensity profiles for the blend of (0.3:0.7) PS/PVME thin film, $0.098 \mu \mathrm{m}$ thick, are displayed. The so-called spinodal ring is clearly visible in Fig. 7(a). Judging by the appearance of the ring only, it would seem that this is a case of typical spinodal decomposition in the 3-dimensional system. However, the intensity in the $q$ region, above the scattering peaks, shows the (-3) power of $q$ dependence (Fig. 7(b)). This is in contrast to the results obtained on a polyelectrolyte blend as described above (Fig. 6), and is characteristic for the Porod's law in the 2-dimensional system. In fact, flat domains formed on the surface of the substrate are observed by an atomic force microscope (AFM) (not shown). The detailed description of this topic can be found elsewhere (Ogawa et al., 2008).

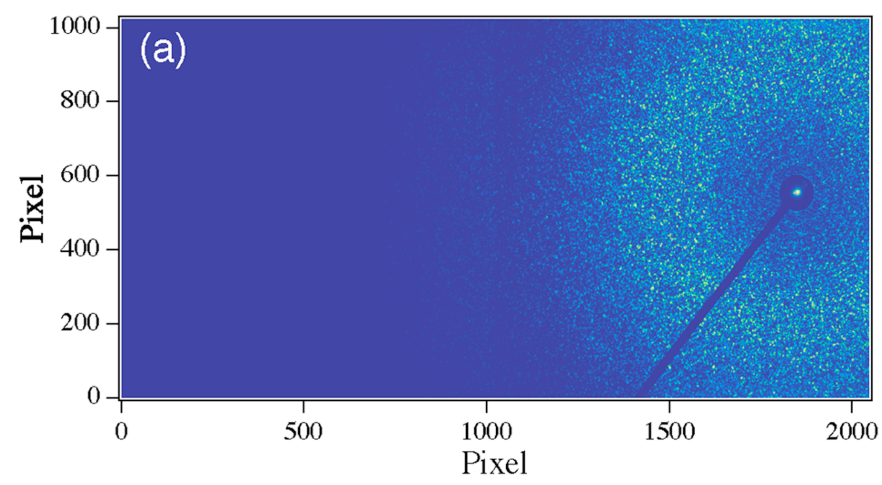

(b)

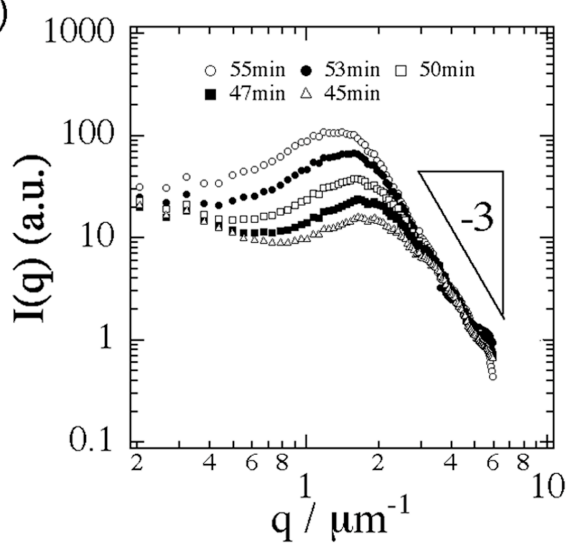

Figure 7 (a): A snapshot ( $t=55 \mathrm{~min}$ ) of two-dimensional scattering image after heating up to $115^{\circ} \mathrm{C}$ taken for $\mathrm{CL}=130 \mathrm{~mm}$ (one pixel corresponds to $48 \mu \mathrm{m}$ ), and (b): the time-resolved onedimensional intensity profiles for the blend of $(0.3: 0.7)$ PS/PVME thin film $0.098 \mu \mathrm{m}$ thick. 


\section{Conclusions}

A SALS instrument with a high resolution at low angles and a high signal-to-noise ratio has been developed aiming at soft matter study. A CMOS image sensor having a large exposure area as the detector has provided us a wide range of scattering vector without using a condenser lens. In summary we have shown that our SALS instrument is suitable for investigation of phenomena, especially described by the characteristic power laws, occurring in solutions and very thin films.

Acknowledgements This work was supported by KAKENHI (Grant-in-Aid for Scientific Research) on Priority Area "Soft Matter Physics" from the Ministry of Education, Culture, Sports, Science and Technology of Japan. We would like to thank Dr B. Gabrys, University of Oxford, for critical reading of the final version of the manuscript. 


\section{References}

Chu, B. (1991) Laser Light Scattering: Basic Principles and Practice. 2nd ed., Boston: Academic Press. Debye, P. (1947). J. Phys. and Colloid Chem. 51, 18-32.

Debye, P. (1946). J. App. Phys. 17, 392-398.

Ferri, F. (1997). Rev. Sci. Instrum. 68, 2265-2273.

Fukushima, H., Ogino, Y., Matsuba, G., Nishida, K. \& Kanaya, T. (2005). Polymer, 46, 1878-1885.

Hashimoto, T., Kumaki, J. \& Kawai, H. (1983). Macromolecules, 16, 641-648.

van de Hulst, H. C. (1957). Light Scattering by Small Particles. New York: John Wiley \& Sons.

Kawai, T. \& Strobl, G. (2004). Macromolecules, 37, 2249-2255.

Nishida, K., Shibata, M., Kanaya, T. \& Kaji, K. (2001). Polymer, 42, 1501-1505.

Ogawa, H., Kanaya, T., Nishida, K. \& Matsuba, G. Polymer, 49, 254-262.

Okada, T., Saito, H. \& Inoue, T. (1992). Macromolecules, 25, 1908-1911.

Plaza, A. \& Stein, R. S. (1959). J. Polym. Sci. 40, 267-270.

Samuels, R. J. (1966). J. Polym. Sci.: Part C, 13, 37-53.

Stein, R. S. \& Rhodes, M. B. (1960). J. App. Phys. 31, 1873-1884.

Tsubouchi, T., Nishida, K. \& Kanaya, T. (2007). Colloids and Surfaces B: Biointerfaces, 56, 265-269.

Zimm, B. H. (1948). J. Chem. Phys. 16, 1093-1099.

Zimm, B. H. (1948). J. Chem. Phys. 16, 1099-1116. 